\title{
Tomography of a Mode-Tunable Coherent Single-Photon Subtractor
}

\author{
Young-Sik Ra, ${ }^{*}$ Clément Jacquard, Adrien Dufour, Claude Fabre, and Nicolas Treps \\ Laboratoire Kastler Brossel, UPMC-Sorbonne Universités, CNRS, ENS-PSL Research University, \\ Collège de France, 4 place Jussieu, 75252 Paris, France \\ (Received 7 February 2017; revised manuscript received 18 April 2017; published 19 July 2017)
}

\begin{abstract}
Single-photon subtraction plays important roles in optical quantum information processing as it provides a non-Gaussian characteristic in continuous-variable quantum information. While the conventional way of implementing single-photon subtraction based on a low-reflectance beam splitter works properly for a single-mode quantum state, it is unsuitable for a multimode quantum state because a single photon is subtracted from all multiple modes without maintaining their mode coherence. Here, we experimentally implement and characterize a mode-tunable coherent single-photon subtractor based on sum-frequency generation. It can subtract a single photon exclusively from one desired time-frequency mode of light or from a coherent superposition of multiple time-frequency modes. To experimentally characterize the time-frequency modes of the single-photon subtractor, we employ quantum process tomography based on coherent states. The mode-tunable coherent single-photon subtractor will be an essential element for realizing non-Gaussian quantum networks necessary to get a quantum advantage in information processing.
\end{abstract}

DOI: 10.1103/PhysRevX.7.031012

\section{INTRODUCTION}

Optical quantum information processing can be classified mainly into two approaches depending on the encoding of quantum information: One is based on continuous electric-field quadratures (thus referred to as continuous-variable quantum information), and the other is based on discrete photon numbers (discrete-variable quantum information). Each of the approaches has its own advantages compared with the other: e.g., in the continuous-variable approach, highly multimode entangled states can be deterministically generated [1-5], and in the discrete-variable approach, quantum processes that cannot be classically simulated can be implemented [6-9]. Therefore, a new approach to combine both advantages has attracted much attention; it is called hybrid quantum information processing [10]. One of the fundamental operations for the hybrid approach is single-photon subtraction, mathematically described by the annihilation operator $\hat{a}$. It introduces a non-Gaussian characteristic (i.e., negativity of the Wigner function) in continuousvariable quantum information [11], which plays essential roles in various quantum information processing, e.g., universal $[12,13]$ and genuine $[14,15]$ quantum computing,

*youngsikra@gmail.com

Published by the American Physical Society under the terms of the Creative Commons Attribution 4.0 International license. Further distribution of this work must maintain attribution to the author(s) and the published article's title, journal citation, and DOI.
Subject Areas: Photonics, Quantum Physics,

Quantum Information preparation of coherent-state-superposition [16-19] and hybrid entanglement [20,21], noiseless linear amplification [22], and entanglement concentration [23,24].

The conventional way of implementing the singlephoton subtraction is to detect a single photon tapped off from an input light using a low-reflectance beam splitter (BS) $[11,25]$. Such a method works well for a single-mode state $[16,17,19]$, but it is unsuitable for a multimode state because the detected photon comes from any mode in an incoherent way, which results in a complete mixture of annihilation operators over the multiple modes [26]. To fully benefit from the highly multimode entangled states available in the continuous variable approach [1-5], one accordingly requires a single-photon subtraction that is able to operate only in the desired modes by maintaining their mode coherence $[18,23,27]$.

In this work, we implement and characterize a singlephoton subtractor which can be tuned to subtract a single photon exclusively from one desired time-frequency mode of light or coherently from multiple time-frequency modes. The single-photon subtractor is based on the detection of a single photon generated via a sum-frequency interaction between an input beam and a strong gate beam in which the choice of the gate-beam modes determines the timefrequency modes of single-photon subtraction [28-31]. To characterize single-photon subtractions with various choices of the gate beam modes, we measure the subtraction matrix of each single-photon subtraction by employing coherent-state quantum process tomography [32,33]: the subtraction matrix contains complete information about a general single-photon subtraction (i.e., amplitude, phase, and 
coherence between different modes) and can be used to quantify its performances. We furthermore discuss the possible experimental imperfections in a single-photon subtractor such as unwanted heralding events (e.g., dark counts or two-photon detection) and optical losses, and estimate their effect on preparing a non-Gaussian quantum state.

\section{DESCRIPTION OF GENERAL SINGLE-PHOTON SUBTRACTION}

We start by introducing a formalism describing general single-photon subtraction in multiple modes [26]. In a single-mode case, single-photon subtraction is uniquely defined by the single-photon annihilation operator $\hat{a}$, which lowers one excitation of a photon-number state $|n\rangle: \hat{a}|n\rangle=$ $\sqrt{n}|n-1\rangle$. This operation is intrinsically nondeterministic (i.e., non-trace-preserving) [34], which succeeds only if a desired outcome is obtained by measuring an ancillary system $[11,25]$. In the multimode case, on the other hand, single-photon subtraction can be diverse because it can consist of, for example, one annihilation operator from multiple modes or several annihilation operators from multiple modes, added as a superposition or as a mixture. In general, single-photon subtraction can be described by a mixture of annihilation operators $\hat{A}_{n}$ with weights $p_{n}$, as shown in Fig. 1, where $\hat{A}_{n}$ can be expressed as a linear combination of basis annihilation operators $\left\{\hat{a}_{0}, \hat{a}_{1}, \ldots\right.$, $\left.\hat{a}_{d-1}\right\}$ in a $d$-dimensional orthonormal mode basis: $\hat{A}_{n}=\sum_{i=0}^{d-1} c_{n i} \hat{a}_{i}$. The bosonic commutation relation of each annihilation operator $\left[\hat{A}_{n}, \hat{A}_{n}^{\dagger}\right]=1$ dictates that $\sum_{i}\left|c_{n i}\right|^{2}=1$, but different annihilation operators are not necessarily orthogonal, $\left[\hat{A}_{n}, \hat{A}_{m}^{\dagger}\right] \neq 0$. A single-photon subtraction $\mathcal{S}$ acting on an input state $\hat{\rho}$ can then be expressed as a quantum map

$$
\mathcal{S}[\hat{\rho}]=\sum_{n} p_{n} \hat{A}_{n} \hat{\rho} \hat{A}_{n}^{\dagger}=\sum_{i, j=0}^{d-1} \chi_{i j} \hat{a}_{i} \hat{\rho} \hat{a}_{j}^{\dagger},
$$

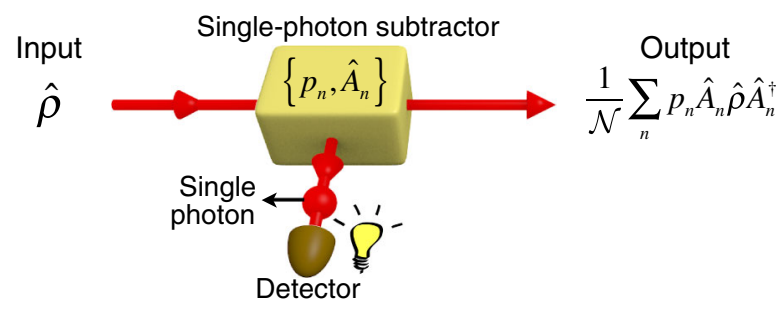

FIG. 1. A single-photon subtractor removes exactly one photon from an input state $\hat{\rho}$, which is heralded by the detection of a single photon in the ancillary path. Single-photon subtraction can be described, in general, by a mixture of annihilation operators $\hat{A}_{0}, \hat{A}_{1}, \ldots$, with the corresponding weights $p_{0}, p_{1}, \ldots$, where all weights sum to 1 , and different annihilation operators are not necessarily orthogonal, $\left[\hat{A}_{n}, \hat{A}_{m}^{\dagger}\right] \neq 0$. The normalization constant $\mathcal{N}$ is $\sum_{n} p_{n}\left\langle\hat{A}_{n}^{\dagger} \hat{A}_{n}\right\rangle$, which is proportional to the heralding probability. where $\chi_{i j}=\sum_{n} p_{n} c_{n i} c_{n j}^{*}$. It results in the output state $\mathcal{S}[\hat{\rho}] / \operatorname{tr}(\mathcal{S}[\hat{\rho}])$ with success probability proportional to $\operatorname{tr}(\mathcal{S}[\hat{\rho}])=\sum_{i j}^{d-1} \chi_{i j}\left\langle\hat{a}_{j}^{\dagger} \hat{a}_{i}\right\rangle$. This formalism can also be obtained from single-photon subtraction based on a multimode beam splitter as reported in Ref. [26]. It is important to note that a single-photon subtraction $\mathcal{S}$ is uniquely determined by the subtraction matrix $\chi$, which is analogous to the density matrix representation for a quantum state. The subtraction matrix is Hermitian and positive semidefinite with a trace of 1 , and $\operatorname{tr}\left(\chi^{2}\right)$ quantifies the purity of the operation, $1 / \operatorname{tr}\left(\chi^{2}\right)$ the effective number of orthogonal modes, and $(\operatorname{tr} \sqrt{\sqrt{\chi} \mu \sqrt{\chi}})^{2}$ the fidelity between two singlephoton subtractions described by $\chi$ and $\mu$.

As an example, a single-photon subtractor based on the conventional method [11,25] makes a completely incoherent single-photon subtraction $\mathcal{S}^{\text {(incoh) }}[\hat{\rho}]=$ $\sum_{i=0}^{d-1}(1 / d) \hat{a}_{i} \hat{\rho} \hat{a}_{i}^{\dagger}$, which gives rise to the identity subtraction matrix $\chi_{i j}^{\text {(incoh) }}=\delta_{i j} / d$ exhibiting purity of $1 / d$. On the other hand, a coherent single-photon subtraction $\mathcal{S}^{(\text {coh })}[\hat{\rho}]=\hat{A}_{0} \hat{\rho} \hat{A}_{0}^{\dagger}$ with $\hat{A}_{0}=\sum_{i=0}^{d-1} c_{i} \hat{a}_{i}$ shows the subtraction matrix of $\chi_{i j}^{(\mathrm{coh})}=c_{i} c_{j}^{*}$ exhibiting purity of 1 . Differently from the incoherent case, the subtraction matrix of a coherent single-photon subtraction contains nonzero off-diagonal elements $\chi_{i j}^{(\mathrm{coh})} \neq 0$ for $i \neq j$, which indicates coherence of single-photon subtraction between different modes.

\section{TOMOGRAPHY OF SINGLE-PHOTON SUBTRACTION}

To experimentally characterize single-photon subtraction, we employ coherent-state quantum process tomography $[32,33]$. As an arbitrary quantum state can be expressed in terms of coherent states (the Glauber-Sudarshan $P$ function) $[35,36]$, any quantum process can be completely characterized by measuring the responses (the output state and the success probability) on various input coherent states. In general, however, characterizing a multimode process requires a large number of coherent states, which grows exponentially with the number of modes [33]. For singlephoton subtraction, on the other hand, the difficulty of multimode characterization can be circumvented because a coherent state is an eigenstate of any annihilation operator [37]; i.e., it is not altered by single-photon subtraction. This fact implies that one can get enough information about single-photon subtraction by measuring only the success probability without measuring the output state. Let us consider the tensor product of coherent states in different modes, $|\beta\rangle=\left|\beta_{0}\right\rangle_{0}\left|\beta_{1}\right\rangle_{1} \ldots\left|\beta_{d-1}\right\rangle_{d-1}$, where the ket subscript denotes a mode for the basis annihilation operators in Eq. (1), and $|\beta|^{2}=\sum_{i=0}^{d-1}\left|\beta_{i}\right|^{2}$ is the average photon number. When it is used as an input state of single-photon subtraction in Eq. (1), the output state becomes the same as the input 
state, and the success probability is proportional to $\sum_{i, j=0}^{d-1} \chi_{i j} \beta_{i} \beta_{j}^{*}$. Note that the success probability is independent of the global phase of $|\beta\rangle$. As the success probability depends on the subtraction matrix $\chi$, its elements $\chi_{i j}$ can be obtained by measuring the probabilities for various input coherent states: We use a coherent state only in the $i$ th mode, $|\beta\rangle_{i}$, to interrogate diagonal elements $\chi_{i i}$, and coherent states only in $i$ and $j$ modes, $\left|\sqrt{\frac{1}{2}} \beta\right\rangle_{i}\left|\sqrt{\frac{1}{2}} \beta\right\rangle_{j}$ (0-phase difference) and $\left|\sqrt{\frac{1}{2}} \beta\right\rangle_{i}\left|\sqrt{-\frac{1}{2}} \beta\right\rangle_{j}(\pi / 2$-phase difference), to obtain the real and imaginary values of off-diagonal elements $\chi_{i j}=\chi_{j i}^{*}$, respectively. The total number of the measurements is $d^{2}$. Note that it is not necessary to investigate the subtraction matrix by varying the average photon number $|\beta|^{2}$ because the subtraction matrix $\chi$ is independent of the input state.

\section{IMPLEMENTATION OF A MODE-TUNABLE COHERENT SINGLE-PHOTON SUBTRACTOR}

We have implemented a mode-tunable coherent singlephoton subtractor for Hermite-Gaussian (HG) timefrequency modes of an input beam based on nonlinear interaction with a strong gate beam, as described in Fig. 2(a). Inside a second-order nonlinear crystal, photons from the two beams give rise to an up-converted photon via sum-frequency generation (SFG). When the up-converted photon is detected by a single-photon detector (SPD), subtraction of a single photon from the input beam is heralded. In the nonlinear conversion process, the joint spectral amplitude of the input and the up-converted beams is engineered in such a way that the spectral amplitude of the gate beam is directly mapped onto the spectral amplitude of the input beam without affecting the spectral amplitude of the up-converted beam, as shown in Fig. 2(b). Such a spectral engineering is accomplished by the horizontal alignment of the joint spectral amplitude and by having a much narrower bandwidth for the up-converted beam with respect to the one of the gate beam; the former condition is satisfied by group velocity matching between the input and the gate beams [28,30], and the latter condition is satisfied by narrow bandwidth phase matching via a thick crystal and/or by narrow bandpass filtering of the up-converted beam. We can therefore tune the timefrequency modes of the single-photon subtraction by controlling the gate beam: If the gate is in the $i$ th $\mathrm{HG}$ mode, a single photon is subtracted from the same $i$ th HG mode. In addition, if the gate is in a superposition of different HG modes, a single photon is subtracted coherently from those HG modes because the spectral amplitude of the up-converted beam is independent of the spectral amplitude of the gate beam [see Fig. 2(b)].

Let us assume that the gate beam is a strong coherent state $|\gamma\rangle=\left|\gamma_{0}\right\rangle_{0}\left|\gamma_{1}\right\rangle_{1} \ldots\left|\gamma_{d-1}\right\rangle_{d-1}$, where the average photon number $|\gamma|^{2}\left(=\sum_{i=0}^{d-1}\left|\gamma_{i}\right|^{2}\right) \gg 1$. Detection of an up-converted photon heralds the coherent single-photon (a)
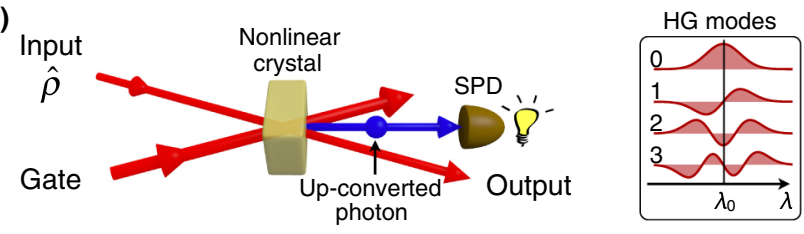

(b)

(c)
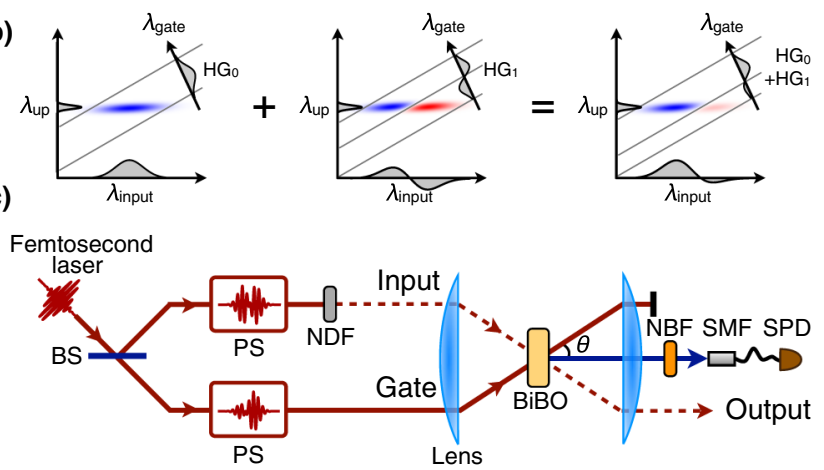

FIG. 2. A mode-tunable coherent single-photon subtractor. (a) Conceptual sketch. Detection of the up-converted photon heralds a single-photon subtraction in the input beam, whose time-frequency modes are determined by the spectral amplitude of the strong gate beam. To characterize the single-photon subtraction, weak coherent states are used as the input. Inset: First four HG time-frequency modes, expressed in the wavelength domain. (b) Joint spectral amplitudes of the input and the up-converted beams with $\mathrm{HG}_{0}$ and $\mathrm{HG}_{1}$ gates. The joint spectral amplitudes can be decomposed into the product of the spectral amplitudes of the input and the up-converted beams, colored in gray. As $\mathrm{HG}_{0}$ and $\mathrm{HG}_{1}$ gate beams give rise to the same spectral amplitude for the up-converted beam, the sum of the joint spectral amplitudes by $\mathrm{HG}_{0}$ and $\mathrm{HG}_{1}$ gates can also be decomposed into the product of the spectral amplitudes of the input and the upconverted beams. (c) Experimental setup: $\theta=2.5^{\circ}$, single-photon detector (SPD), nonpolarizing BS, pulse shaper (PS), neutral density filter (NDF), narrow bandpass filter (NBF), and singlemode fiber (SMF).

subtraction $\mathcal{S}^{(\mathrm{coh})}[\hat{\rho}]=\hat{A}_{0} \hat{\rho} \hat{A}_{0}^{\dagger}$ with $\hat{A}_{0}=\sum_{i=0}^{d-1}(-1)^{i} c_{i} \hat{a}_{i}^{(\mathrm{HG})}$, where $\hat{a}_{i}^{(\mathrm{HG})}$ is the annihilation operator for the $i$ th HG mode, and $c_{i}\left(=\gamma_{i} /|\gamma|\right)$ is the normalized coefficient. The additional coefficient $(-1)^{i}$ originates from the wavelength inversion with respect to the central wavelength by energy conservation of SFG [38], which makes the sign change only for antisymmetric HG modes. In practice, the singlephoton subtraction can entail additional annihilation operators $\hat{A}_{n(\neq 0)}$ (e.g., due to a nonideal joint spectral amplitude):

$$
\mathcal{S}^{(\mathrm{SFG})}[\hat{\rho}]=p_{0} \hat{A}_{0} \hat{\rho} \hat{A}_{0}^{\dagger}+\sum_{n=1} p_{n} \hat{A}_{n} \hat{\rho} \hat{A}_{n}^{\dagger}
$$

with $\sum_{n=0} p_{n}=1$. The weight of $\hat{A}_{0}$, i.e., $p_{0}$, is defined as mode selectivity of single-photon subtraction [39], which becomes unity for the ideal case. 
Figure 2(c) describes the experimental setup developed to implement and characterize the mode-tunable coherent single-photon subtractor. A femtosecond laser (central wavelength, $795 \mathrm{~nm}$; full width at half maximum (FWHM), $11 \mathrm{~nm}$; repetition rate, $76 \mathrm{MHz}$ ) is split into input and gate beams at a BS. The spectral amplitudes of the two beams are individually controlled by PS having a spectral resolution of $0.2 \mathrm{~nm}$. A NDF attenuates the input beam to prepare a coherent state, having the average photon number of one per pulse, and the gate beam has $1 \mathrm{~mW}$ power (corresponding to around $5 \times 10^{7}$ photons per pulse). The two beams (beam diameter: $1.6 \mathrm{~mm}$ ) are focused by a single plano-convex lens (focal length: $190 \mathrm{~mm}$ ) onto a bismuth borate $(\mathrm{BiBO})$ bulk crystal (thickness: $2.5 \mathrm{~mm}$ ), which generates frequency upconverted light (central wavelength: $397.5 \mathrm{~nm}$; FWHM: $0.6 \mathrm{~nm}$ ) via SFG. The phase difference between the beams need not be fixed as it does not affect the operation of the single-photon subtractor. To achieve a high mode selectivity, the group velocities of the beams inside the crystal are matched by using the same central wavelength and the same polarization [30], and a narrow bandwidth of the up-converted light is generated by a phase matching via the thick crystal, which is further filtered by a narrow bandpass filter (FWHM: $0.4 \mathrm{~nm}$ ). The up-converted light is then collected into a single mode fiber, and it is detected by an on-off type SPD (Hamamatsu C13001-01; quantum efficiency: $40 \%$; dark count rate: $10 \mathrm{~Hz}$ ). To measure the success probability of single-photon subtraction for the quantum process tomography, we record the count rates of the SPD with various input coherent states as described in Sec. III.

\section{EXPERIMENTAL RESULTS}

We start by implementing the single-photon subtraction for the $\mathrm{HG}_{0}$ mode by sending a gate beam in the $\mathrm{HG}_{0}$ mode (central wavelength: $795 \mathrm{~nm}$; FWHM: $4 \mathrm{~nm}$ ). To represent its subtraction matrix, we choose a wavelength-band mode basis, which consists of 25 different wavelength bands from $786 \mathrm{~nm}$ to $804 \mathrm{~nm}$ (see Ref. [40] for their spectrums). We characterize the implemented subtraction by using input coherent states to the number of 625 in the wavelengthband modes, and the average photon number of the input coherent states is increased up to 90 for fast data acquisition. To construct a physical subtraction matrix (positive and semidefinite), we have employed the maximum likelihood technique [41] for all the following tomography results. Figure 3(a) shows the obtained subtraction matrix by using a $\mathrm{HG}_{0}$ gate beam. Note that not only diagonal terms but also off-diagonal terms exist around a 795-nm wavelength, manifesting coherent single-photon subtraction from different wavelength-band modes; the imaginary part of the matrix shows negligibly small values because the phase is almost zero over all the wavelength-band modes. The dominant eigenvalue of the subtraction matrix, obtained via diagonalization, corresponds to the mode selectivity $p_{0}$. Since this value is close to 1 , we can associate the corresponding eigenvector with the dominant single-photon annihilation operator $\hat{A}_{0}$. This is shown in the last row of Fig. 3(a), which agrees well with the spectral amplitude of the gate beam and shows a high mode selectivity and purity [42].

We next tune the single-photon subtractor by adjusting the spectral amplitude of the gate beam. Figure 3(b) shows
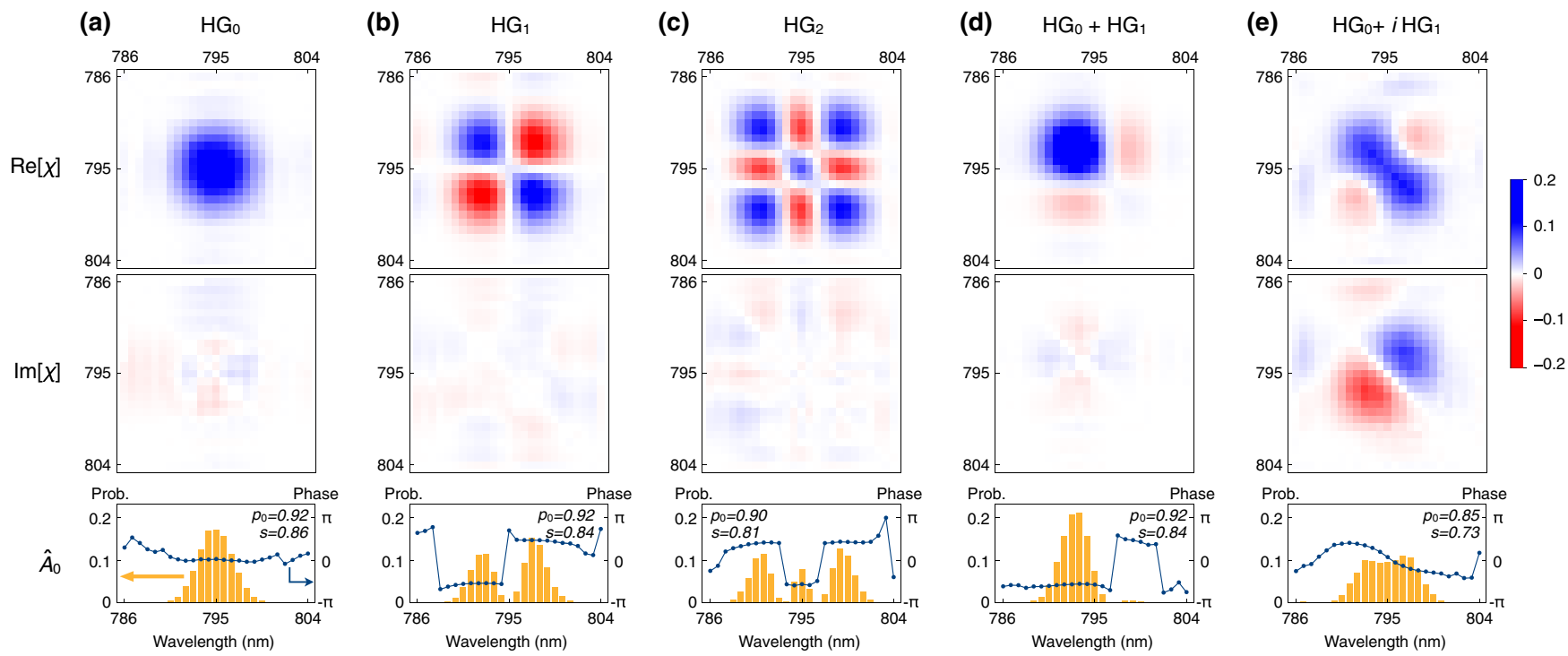

FIG. 3. Tomography of single-photon subtraction based on 25 wavelength-band modes. The first, second, and third rows are real and imaginary parts of the subtraction matrix $\chi$, and the mode of the dominant annihilation operator $\hat{A}_{0}$, respectively. In the third row, bars and points represent the probability and phase of each wavelength band, respectively, and the line is a visual guide. Note that $p_{0}$ is mode selectivity, and $s$ is purity. 
the subtraction matrix obtained by using a $\mathrm{HG}_{1}$ gate beam. The two negative areas in the real part are due to the sign difference of the $\mathrm{HG}_{1}$ mode with respect to the central wavelength [see the inset of Fig. 2(a)]; this also confirms the coherence between the longer and the shorter wavelength parts. The sign change also appears as the $\pi$-phase jump in the dominant annihilation operator $\hat{A}_{0}$, shown in the last row of Fig. 3(b). Similarly, we implement and characterize single-photon subtraction for the $\mathrm{HG}_{2}$ mode, shown in Fig. 3(c). Figures 3(d) and 3(e) are obtained by sending a gate beam in a superposition of $\mathrm{HG}_{0}$ and $\mathrm{HG}_{1}$ modes, (d) with 0-phase difference and (e) with $\pi / 2$-phase difference. As $\mathrm{HG}_{1}$ implements $-\hat{a}_{1}^{(\mathrm{HG})}$, as discussed in Sec. IV, the sum of $\mathrm{HG}_{0}$ and $\mathrm{HG}_{1}$ gate modes with 0 -phase difference implements $(1 / \sqrt{2})\left(\hat{a}_{0}^{(\mathrm{HG})}-\hat{a}_{1}^{(\mathrm{HG})}\right)$, which makes the subtraction matrix distributed at lower wavelengths than the central wavelength. The $\pi / 2$-phase difference between the gate modes results in imaginary values in the subtraction matrix because of the phase difference between wavelength-band modes. We provide additional subtraction matrices using different gate beams in Ref. [40].

As our single-photon subtractor is designed for parametric multimode sources $[3,43]$, we now characterize it with a mode basis approximating the eigenmodes of this process: $\mathrm{HG}$ modes $\left\{\mathrm{HG}_{0}, \mathrm{HG}_{1}, \ldots, \mathrm{HG}_{6}\right\}$. The input and the gate beams are based on the same HG-mode basis (central wavelength: $795 \mathrm{~nm}$; FWHM of $\mathrm{HG}_{0}$ : $4 \mathrm{~nm}$ ), whose measured spectrums are provided in Ref. [40]. For the characterization, we use up to 49 input coherent states by maintaining the average photon number of one per pulse. For a $\mathrm{HG}_{0}$ gate beam, the subtraction matrix, shown in Fig. 4(a), has its dominant element in the $\mathrm{HG}_{0}$ mode. It also exhibits high fidelity with the ideal operation $\hat{a}_{0}^{(\mathrm{HG})}$ as well as a high mode selectivity and purity. As the gate mode is shifted to higher order, the dominant element in the subtraction matrix is shifted accordingly [see Fig. 4(b) and Ref. [40]]. When the gate beam is in a superposition of $\mathrm{HG}_{0}$ and $\mathrm{HG}_{1}$, a coherent single-photon subtraction takes place, as shown in Fig. 4(c) for the same phase and Fig. 4(d) for $\pi / 2$-phase difference between the two HG modes. The off-diagonal elements between $\mathrm{HG}_{0}$ and $\mathrm{HG}_{1}$ modes clearly show the coherence between $\hat{a}_{0}^{(\mathrm{HG})}$ and $\hat{a}_{1}^{(\mathrm{HG})}$ and the tunability of their relative phase. The fidelities with the ideal operations $(1 / \sqrt{2})\left(\hat{a}_{0}^{(\mathrm{HG})}-\hat{a}_{1}^{(\mathrm{HG})}\right)$ and $(1 / \sqrt{2})\left(\hat{a}_{0}^{(\mathrm{HG})}-i \hat{a}_{1}^{(\mathrm{HG})}\right)$, respectively, are also high. The single-photon subtractor can also be tuned to act on multiple HG modes coherently, $(1 / \sqrt{5}) \sum_{i=0}^{4}(-1)^{i} \hat{a}_{i}^{(\mathrm{HG})}$ in Fig. 4(e) and $(1 / \sqrt{7}) \sum_{i=0}^{6}(-1)^{i} \hat{a}_{i}^{(\mathrm{HG})}$ in Fig. 4(f), respectively. To investigate the independence of the subtraction matrix on the input state, we characterize the single-photon subtraction for Fig. 4(f) using input coherent states with much higher average photon number amounting to 90. The obtained matrix, shown in Fig. 4(g), is almost identical to the subtraction matrix measured by an average (a) $\mathrm{HG}_{0}$

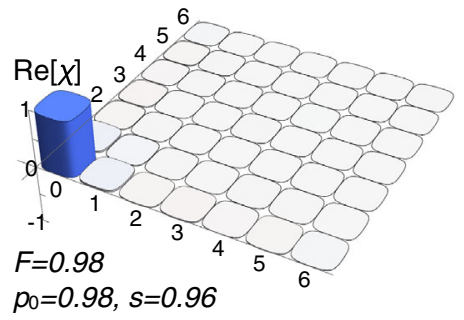

(e) $\mathrm{HG}_{0}+\ldots+\mathrm{HG}_{4}$

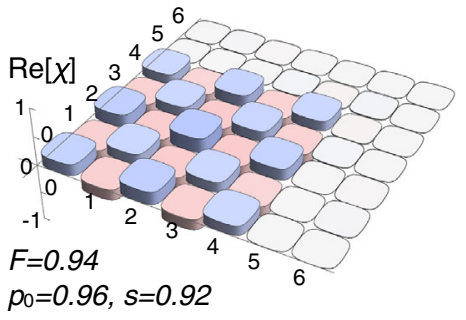

(b) $\mathrm{HG}_{1}$

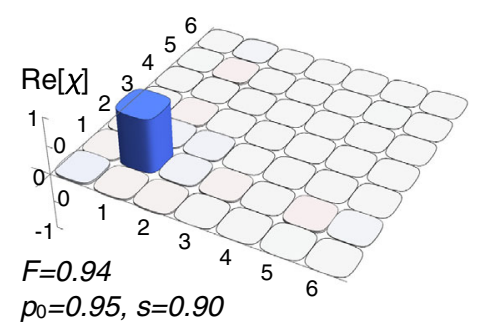

(f) $\mathrm{HG}_{0}+\ldots+\mathrm{HG}_{6}$

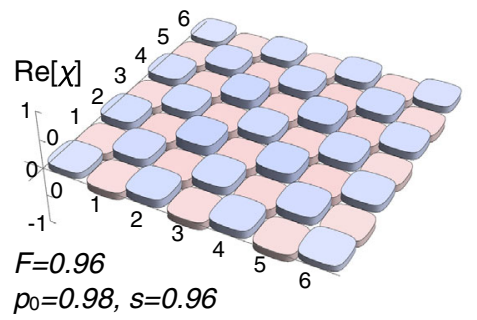

(c) $\mathrm{HG}_{0}+\mathrm{HG}_{1}$
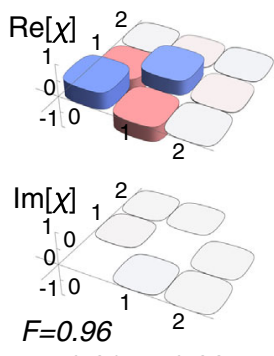

$p_{0}=0.96, s=0.93$ (d) $\mathrm{HG}_{0}+i \mathrm{HG}_{1}$
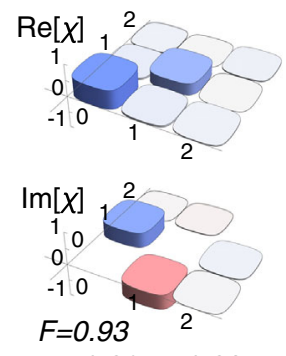

$p_{0}=0.96, s=0.92$

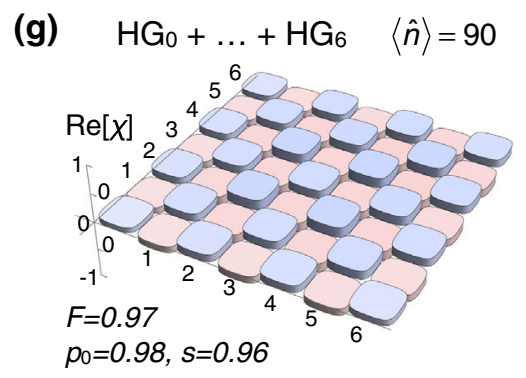

FIG. 4. Tomography of single-photon subtraction based on seven HG modes. An index in the horizontal plane denotes the order of a HG mode from 0 to 6 . In diagrams ( $\mathrm{a}, \mathrm{b}, \mathrm{e}, \mathrm{f}, \mathrm{g}$ ), we present only the real part of the subtraction matrix $\chi$ as the imaginary part is negligibly small. For the same reason, we present only a truncated part of a matrix in diagrams (c) and (d). The average photon number of the probe beam is 1 in diagrams (a-f) and 90 in diagram $(\mathrm{g})$. Note that $F$ is fidelity with the ideal subtraction matrix, $p_{0}$ is mode selectivity, and $s$ is purity. See Ref. [40] for the full data. 
photon number of 1 in Fig. 4(f), exhibiting fidelity of 0.99 between them. We provide additional subtraction matrices using different gate beams in Ref. [40].

\section{DISCUSSION}

We discuss here the possible imperfections of the singlephoton subtractor by taking into account undesired heralding events. In practice, a single click by an on-off SPD does not always herald single-photon subtraction because it may originate from an accidental event by detector dark counts or detection of two photons [25]. A realistic single-photon subtraction $\mathcal{R}$ is then described as

$$
\mathcal{R}[\hat{\rho}]=w_{0} \hat{\rho}+w_{1} \mathcal{S}[\hat{\rho}]+w_{2} \mathcal{S}[\mathcal{S}[\hat{\rho}]],
$$

which results in the output state $\mathcal{R}[\hat{\rho}] / \operatorname{tr}(\mathcal{R}[\hat{\rho}])$ with the success probability proportional to $\operatorname{tr}(\mathcal{R}[\hat{\rho}])$. The first term represents the identity operation due to an accidental click, the middle term is the desired single-photon subtraction $\mathcal{S}$ in Eq. (1), and the last term is the double application of the single-photon subtraction due to two-photon detection. Therefore, their respective weights $w_{0}, w_{1}$, and $w_{2}(=1-$ $\left.w_{0}-w_{1}\right)$ are an important factor to assess the quality of the single-photon subtractor. These weights can be measured using input coherent states. If a coherent state $|\beta\rangle_{0}$ in the dominant subtraction mode is used, the success probability of the operation is proportional to $\operatorname{tr}(\mathcal{R}[\hat{\rho}])=w_{0}+$ $w_{1} p_{0}|\beta|^{2}+w_{2} p_{0}^{2}|\beta|^{4}$; thus, measuring the success probability with respect to $|\beta|^{2}$ can reveal the weights $w_{0}, w_{1}$, and $w_{2}$. Note that the mode selectivity $p_{0}$ can be obtained through the tomography method presented in Secs. III and V. The implemented single-photon subtractor exhibits a dominating contribution of single-photon subtraction $\left(w_{1}=0.99\right)$, a very small contribution of the identity operation $\left(w_{0}=0.01\right)$, and negligible two-photon subtraction $\left(w_{2}<10^{-3}\right)$ (see Ref. [40] for the experimental data). The significant suppression of two-photon subtraction is due to a low conversion ratio $\left(10^{-3}\right)$ of the input beam to the up-converted beam for a $1-\mathrm{mW}$ gate beam, which still provides a moderate heralding rate of around $2 \mathrm{kHz}$, with an input state with an average photon number of 1 .

Based on this realistic model of single-photon subtraction, we can estimate its performance (e.g., negativity of the Wigner function) in a general experimental condition including losses. Figure 5 depicts the sequence of operations: Initial loss $\mathcal{L}^{\text {(in) }}$ accounts for imperfection of quantum state preparation (e.g., excess noise of squeezed vacuum) and the propagation loss before single-photon subtraction, and final loss $\mathcal{L}^{(\mathrm{fi})}$ accounts for the propagation loss after single-photon subtraction and the inefficiency of quantum state measurement (e.g., homodyne detection). Such optical losses can be modeled as a coupling with vacuum by a fictitious beam splitter $\mathcal{B}_{T}$ (transmittance: $T$ ). For simplicity, let us consider homogeneous loss for all the

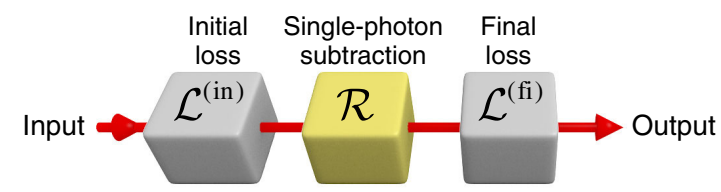

FIG. 5. The input state sequentially experiences initial loss $\mathcal{L}^{(\text {in) }}$, realistic single-photon subtraction $\mathcal{R}$, and final loss $\mathcal{L}^{(\mathrm{fi})}$.

modes, $\quad \mathcal{L}^{(\text {in })}=\mathcal{B}_{T^{(\text {in })}} \otimes \mathcal{B}_{T^{(\text {in })}} \otimes \ldots$ and $\quad \mathcal{L}^{(\mathrm{fi})}=\mathcal{B}_{T^{(\mathrm{i})}} \otimes$ $\mathcal{B}_{T^{(\mathrm{fi})}} \otimes \ldots$, where $T^{(\mathrm{in})}\left(T^{(\mathrm{fi})}\right)$ is the transmittance of a fictitious beam splitter for initial (final) loss. If the input state is a multimode state $\hat{\rho}=\hat{\sigma} \otimes \hat{\sigma} \otimes \ldots$, which consists of identically squeezed vacuum $\hat{\sigma}$ in each mode [3], the final quantum state reduced to the dominant subtraction mode reads

$$
\begin{aligned}
\hat{\rho}_{0}^{(\mathrm{fi})} & \equiv \frac{\operatorname{tr}_{12 \ldots}\left(\mathcal{L}^{(\mathrm{fii})}\left[\mathcal{R}\left[\mathcal{L}^{(\mathrm{in})}[\hat{\rho}]\right]\right]\right)}{\operatorname{tr}_{012 \ldots}\left(\mathcal{L}^{(\mathrm{fi})}\left[\mathcal{R}\left[\mathcal{L}^{(\mathrm{in})}[\hat{\rho}]\right]\right]\right)} \\
& =r^{(\mathrm{false})} \mathcal{B}_{T^{(\mathrm{ovr})}}[\hat{\sigma}]+r^{(\mathrm{corr})} \frac{\hat{A}_{0} \mathcal{B}_{T^{(\mathrm{ovr})}}[\hat{\sigma}] \hat{A}_{0}^{\dagger}}{\operatorname{tr}\left(\hat{A}_{0} \mathcal{B}_{\left.T^{(\mathrm{ovr})}[\hat{\sigma}] \hat{A}_{0}^{\dagger}\right)}\right.}
\end{aligned}
$$

where $T^{(\mathrm{ovr})}\left(=T^{(\mathrm{fi})} T^{(\mathrm{in})}\right)$ is the overall transmittance of the entire setup, and the two-photon detection weight $w_{2}$ of $\mathcal{R}$ is set to zero, as it is negligible. The first term, $\mathcal{B}_{T^{(\text {ovr })}}[\hat{\sigma}]$, is a squeezed vacuum mixed with the vacuum noise heralded by a false click in $\mathcal{R}$. It originates from the accidental click (quantified by $w_{0}=1-w_{1}$ ) and single-photon click from other modes (quantified by mode selectivity $p_{0}$ ) and has a ratio of $r^{\text {(false) }}=\left\{\left[\left(1-w_{1}\right)+w_{1}\left(1-p_{0}\right) T^{(\text {in })}\langle\hat{n}\rangle_{\hat{\sigma}}\right] /\right.$ $\left.\left.\left[\left(1-w_{1}\right)+w_{1} T^{(\mathrm{in})}\langle\hat{n}\rangle_{\hat{\sigma}}\right)\right]\right\}$, where $\langle\hat{n}\rangle_{\hat{\sigma}}$ is the average photon number of $\hat{\sigma}$. The second term is the single-photon subtracted state from $\mathcal{B}_{T^{(\text {ovr })}}[\hat{\sigma}]$ heralded by a correct click in $\mathcal{R}$, which originates from single-photon subtraction exclusively from the dominant subtraction mode; it has a ratio of $r^{\text {(corr) }}=\left\{\left(w_{1} p_{0} T^{(\mathrm{in})}\langle\hat{n}\rangle_{\hat{\sigma}}\right) /\left[\left(1-w_{1}\right)+w_{1} T^{(\mathrm{in})}\langle\hat{n}\rangle_{\hat{\sigma}}\right]\right\}$. Based on the characteristics of the implemented singlephoton subtractor $\left(w_{1}=0.99, p_{0}=0.9\right)$ and the typical experimental conditions (initial and final losses of $10 \%$, respectively, which incorporate $2 \%$ optical loss of the implemented single-photon subtractor), one can estimate that a non-Gaussian state exhibiting a negativity of the Wigner function amounting to $-(0.3 / 2 \pi)$ can be obtained from an input state of 4-dB multimode squeezed vacua [44].

\section{CONCLUSIONS}

We have experimentally implemented a mode-tunable coherent single-photon subtractor and characterized it by employing coherent-state quantum process tomography. We could readily tune the time-frequency modes of singlephoton subtraction by adjusting the spectral modes of the gate beam, which does not require a physical reconstruction of a mode-coupling device [6-9]. We have implemented 
various single-photon subtractions such as a subtraction for one HG mode and a coherent subtraction for several HG modes. The subtraction matrices obtained in the wavelength-band modes reveal the modes of the single-photon subtractions in the wavelength domain, and the subtraction matrices in the HG modes directly show the coherence between different HG modes, which is required for parametric multimode sources [3,43]. A high mode selectivity (typically larger than 0.9) and low imperfections (dark count contribution around $1 \%$ and optical loss around 2\%) of the single-photon subtractor show its direct applicability to generate multimode non-Gaussian states.

We anticipate that the single-photon subtractor will be an essential operation for a non-Gaussian quantum network, e.g., preparation of hybrid multimode entangled states $[10,20,21]$, distillation of multipartite entanglement [23,27], measurement-based quantum computing [13,45], etc. In addition, our tomography method is not limited to characterize time-frequency modes: it can be generally applied to other types of light modes such as spatial [43], polarization [46], and spatiotemporal [47] modes, and it can be extended to characterize a general multimode quantum process [33]. In particular, it will be useful for identifying couplings among many connected modes (e.g., BosonSampling [6-9] and multiple scattering [48]) and, more importantly, for quantifying the coherence of such connections [49].

\section{ACKNOWLEDGMENTS}

We thank V. Parigi, J. Roslund, C. Silberhorn, and B. Brecht for fruitful discussions. This work is supported by the French National Research Agency projects COMB and SPOCQ, the European Research Council starting grant Frecquam, and the European Union Grant QCUMbER (No. 665148). C. F. and N. T. are members of the Institut Universitaire de France. Y.-S. R. acknowledges support from the European Commission through Marie Skłodowska-Curie actions (Grant Agreement No. 708201).

[1] C. Weedbrook, S. Pirandola, R. García-Patrón, N. J. Cerf, T. C. Ralph, J. H. Shapiro, and S. Lloyd, Gaussian Quantum Information, Rev. Mod. Phys. 84, 621 (2012).

[2] S. Yokoyama, R. Ukai, S. C. Armstrong, C. Sornphiphatphong, T. Kaji, S. Suzuki, J. I. Yoshikawa, H. Yonezawa, N.C. Menicucci, and A. Furusawa, Ultra-Large-Scale Continuous-Variable Cluster States Multiplexed in the Time Domain, Nat. Photonics 7, 982 (2013).

[3] J. Roslund, R. M. de Araújo, S. Jiang, C. Fabre, and N. Treps, Wavelength-Multiplexed Quantum Networks with Ultrafast Frequency Combs, Nat. Photonics 8, 109 (2014).

[4] M. Chen, N. C. Menicucci, and O. Pfister, Experimental Realization of Multipartite Entanglement of 60 Modes of a Quantum Optical Frequency Comb, Phys. Rev. Lett. 112, 120505 (2014).
[5] Y. Cai, J. Roslund, G. Ferrini, F. Arzani, X. Xu, C. Fabre, and N. Treps, Multimode entanglement in reconfigurable graph states using optical frequency combs, Nat. Commun. 8, 15645 (2017).

[6] M. A. Broome, A. Fedrizzi, S. Rahimi-Keshari, J. Dove, S. Aaronson, T. C. Ralph, and A. G. White, Photonic Boson Sampling in a Tunable Circuit, Science 339, 794 (2013).

[7] J. B. Spring, B. J. Metcalf, P. C. Humphreys, W. S. Kolthammer, X. M. Jin, M. Barbieri, A. Datta, N. Thomas-Peter, N. K. Langford, D. Kundys et al., Boson Sampling on a Photonic Chip, Science 339, 798 (2013).

[8] A. Crespi, R. Osellame, R. Ramponi, D. J. Brod, E. F. Galvão, N. Spagnolo, C. Vitelli, E. Maiorino, P. Mataloni, and F. Sciarrino, Integrated Multimode Interferometers with Arbitrary Designs for Photonic Boson Sampling, Nat. Photonics 7, 545 (2013).

[9] M. Tillmann, B. Dakic, R. Heilmann, S. Nolte, A. Szameit, and P. Walther, Experimental Boson Sampling, Nat. Photonics 7, 540 (2013).

[10] U. L. Andersen, J. S. Neergaard-Nielsen, P. Van Loock, and A. Furusawa, Hybrid Discrete- and ContinuousVariable Quantum Information, Nat. Phys. 11, 713 (2015).

[11] J. Wenger, R. Tualle-Brouri, and P. Grangier, Non-Gaussian Statistics from Individual Pulses of Squeezed Light, Phys. Rev. Lett. 92, 153601 (2004).

[12] S. Lloyd and S. L. Braunstein, Quantum Computation over Continuous Variables, Phys. Rev. Lett. 82, 1784 (1999).

[13] N. C. Menicucci, P. Van Loock, M. Gu, C. Weedbrook, T. C. Ralph, and M. A. Nielsen, Universal Quantum Computation with Continuous-Variable Cluster States, Phys. Rev. Lett. 97, 110501 (2006).

[14] S. D. Bartlett, B. C. Sanders, S. L. Braunstein, and K. Nemoto, Efficient Classical Simulation of Continuous Variable Quantum Information Processes, Phys. Rev. Lett. 88, 097904 (2002).

[15] A. Mari and J. Eisert, Positive Wigner Functions Render Classical Simulation of Quantum Computation Efficient, Phys. Rev. Lett. 109, 230503 (2012).

[16] A. Ourjoumtsev, R. Tualle-Brouri, J. Laurat, and P. Grangier, Generating Optical Schrödinger Kittens for Quantum Information Processing, Science 312, 83 (2006).

[17] J. Neergaard-Nielsen, B. Nielsen, C. Hettich, K. Molmer, and E. Polzik, Generation of a Superposition of Odd Photon Number States for Quantum Information Networks, Phys. Rev. Lett. 97, 083604 (2006).

[18] A. Ourjoumtsev, F. Ferreyrol, R. Tualle-Brouri, and P. Grangier, Preparation of Non-local Superpositions of Quasi-classical Light States, Nat. Phys. 5, 189 (2009).

[19] J. S. Neergaard-Nielsen, M. Takeuchi, K. Wakui, H. Takahashi, K. Hayasaka, M. Takeoka, and M. Sasaki, Optical Continuous-Variable Qubit, Phys. Rev. Lett. 105, 053602 (2010).

[20] H. Jeong, A. Zavatta, M. Kang, S. W. Lee, L. S. Costanzo, S. Grandi, T. C. Ralph, and M. Bellini, Generation of Hybrid Entanglement of Light, Nat. Photonics 8, 564 (2014).

[21] O. Morin, K. Huang, J. Liu, H. Le Jeannic, C. Fabre, and J. Laurat, Remote Creation of Hybrid Entanglement between Particle-like and Wave-like Optical Qubits, Nat. Photonics 8, 570 (2014). 
[22] A. Zavatta, J. Fiurášek, and M. Bellini, A High-Fidelity Noiseless Amplifier for Quantum Light States, Nat. Photonics 5, 52 (2011).

[23] A. Ourjoumtsev, A. Dantan, R. Tualle-Brouri, and P. Grangier, Increasing Entanglement between Gaussian States by Coherent Photon Subtraction, Phys. Rev. Lett. 98, 030502 (2007).

[24] H. Takahashi, J. S. Neergaard-Nielsen, M. Takeuchi, M. Takeoka, K. Hayasaka, A. Furusawa, and M. Sasaki, Entanglement Distillation from Gaussian Input States, Nat. Photonics 4, 178 (2010).

[25] M. S. Kim, Recent Developments in Photon-Level Operations on Travelling Light Fields, J. Phys. B 41, 133001 (2008).

[26] V. Averchenko, C. Jacquard, V. Thiel, C. Fabre, and N. Treps, Multimode Theory of Single-Photon Subtraction, New J. Phys. 18, 083042 (2016).

[27] H.-J. Kim, J. Kim, and H. Nha, Enhanced Multipartite Quantum Correlation by Non-Gaussian Operations, Phys. Rev. A 88, 032109 (2013).

[28] A. Eckstein, B. Brecht, and C. Silberhorn, A Quantum Pulse Gate Based on Spectrally Engineered Sum Frequency Generation, Opt. Express 19, 13770 (2011).

[29] B. Brecht, A. Eckstein, R. Ricken, V. Quiring, H. Suche, L. Sansoni, and C. Silberhorn, Demonstration of Coherent Time-Frequency Schmidt Mode Selection Using DispersionEngineered Frequency Conversion, Phys. Rev. A 90, 030302 (2014).

[30] V. A. Averchenko, V. Thiel, and N. Treps, Nonlinear Photon Subtraction from a Multimode Quantum Field, Phys. Rev. A 89, 063808 (2014).

[31] P. Manurkar, N. Jain, M. Silver, Y.-P. Huang, C. Langrock, M. M. Fejer, P. Kumar, and G. S. Kanter, Multidimensional Mode-Separable Frequency Conversion for High-Speed Quantum Communication, Optica 3, 1300 (2016).

[32] M. Lobino, D. Korystov, C. Kupchak, E. Figueroa, B. C. Sanders, and A. I. Lvovsky, Complete Characterization of Quantum-Optical Processes, Science 322, 563 (2008).

[33] I. A. Fedorov, A. K. Fedorov, Y. V. Kurochkin, and A. I. Lvovsky, Tomography of a Multimode Quantum Black Box, New J. Phys. 17, 043063 (2015).

[34] R. Kumar, E. Barrios, C. Kupchak, and A. I. Lvovsky, Experimental Characterization of Bosonic Creation and Annihilation Operators, Phys. Rev. Lett. 110, 130403 (2013).

[35] E. C. G. Sudarshan, Equivalence of Semiclassical and Quantum Mechanical Descriptions of Statistical Light Beams, Phys. Rev. Lett. 10, 277 (1963).
[36] R. J. Glauber, Photon Correlations, Phys. Rev. Lett. 10, 84 (1963).

[37] A. Zavatta, V. Parigi, M. S. Kim, and M. Bellini, Subtracting Photons from Arbitrary Light Fields: Experimental Test of Coherent State Invariance by Single-Photon Annihilation, New J. Phys. 10, 123006 (2008).

[38] We consider the case in which the frequency bandwidth is much smaller than the central frequency.

[39] D. V. Reddy, M. G. Raymer, C. J. McKinstrie, L. Mejling, and K. Rottwitt, Temporal mode selectivity by frequency conversion in second-order nonlinear optical waveguides, Opt. Express 21, 13840 (2013).

[40] See Supplemental Material at http://link.aps.org/ supplemental/10.1103/PhysRevX.7.031012 for additional experimental data.

[41] D. James, P. G. Kwiat, W. J. Munro, and A. White, Measurement of Qubits, Phys. Rev. A 64, 052312 (2001).

[42] Note that the purity of the completely mixed subtraction matrix in 25 modes is 0.04 .

[43] L. Lopez, B. Chalopin, A. R. de la Souchère, C. Fabre, A. Maître, and N. Treps, Multimode Quantum Properties of a Self-Imaging Optical Parametric Oscillator: Squeezed Vacuum and Einstein-Podolsky-Rosen-Beams Generation, Phys. Rev. A 80, 043816 (2009).

[44] In the ideal case, a single-photon subtracted squeezed vacuum state or a single-photon Fock state has a negativity of the Wigner function amounting to $-(1 / 2 \pi)$.

[45] G. Ferrini, J.P. Gazeau, T. Coudreau, C. Fabre, and N. Treps, Compact Gaussian Quantum Computation by Multi-pixel Homodyne Detection, New J. Phys. 15, 093015 (2013).

[46] Y.-S. Ra, H.-T. Lim, and Y.-H. Kim, Remote Preparation of Three-Photon Entangled States via Single-Photon Measurement, Phys. Rev. A 94, 042329 (2016).

[47] A. Gatti, E. Brambilla, L. Caspani, O. Jedrkiewicz, and L. A. Lugiato, XEntanglement: The Nonfactorable Spatiotemporal Structure of Biphoton Correlation, Phys. Rev. Lett. 102, 223601 (2009).

[48] H. Defienne, M. Barbieri, I. A. Walmsley, B. J. Smith, and S. Gigan, Two-Photon Quantum Walk in a Multimode Fiber, Sci. Adv. 2, e1501054 (2016).

[49] M. C. Tichy, Y.-S. Ra, H.-T. Lim, C. Gneiting, Y.-H. Kim, and K. Mølmer, Double-Fock Superposition Interferometry for Differential Diagnosis of Decoherence, New J. Phys. 17, 023008 (2015). 